\title{
La prise en charge de la douleur aux urgences : indicateur de qualité et de performance aux urgences
}

\author{
Managing Pain in Emergency Departments: an Indicator of their Quality and Performance
}

\section{E. Casalino}

Reçu le 19 septembre 2017; accepté le 14 février 2018

(C) SFMU et Lavoisier SAS 2018

Résumé La qualité et la performance des services d'urgences sont des objectifs nationaux. La mise en place d'une stratégie d'amélioration continue de la qualité et de la performance nécessite de fédérer l'ensemble des équipes de soins autour d'un projet innovant. Les visites de certification sont un des outils que nous pouvons utiliser comme un levier pour mettre en place et améliorer la qualité. Un projet fédérateur nécessite de définir des valeurs partagées entre les équipes de soins. La lutte contre la douleur est une des valeurs fortes pour les équipes de soins, notamment aux urgences. Nous devons utiliser le projet de prise en charge de la douleur comme un moyen de revoir nos organisations, d'évaluer nos processus et de les simplifier, afin d'atteindre les objectifs définis par une cartographie des risques spécifiques. Nous présentons ici l'expérience de nos services d'urgences pour la prise en charge de la douleur d'origine traumatique, les méthodes suivies et les outils d'amélioration mis en place.

Mots clés Douleur - Urgences · Amélioration continue de la qualité · Traumatisme

\begin{abstract}
Emergency department's quality and performance improvement are national objectives. The implementation of a strategy for continuous quality and performance improvement requires federating all health care workers around an
\end{abstract}

E. Casalino $(\bowtie)$

Service d'accueil des urgences (SAU),

Assistance publique-Hôpitaux de Paris (AP-HP),

hôpital Bichat-Claude-Bernard,

46, rue Henri-Huchard, F-75018 Paris, France

e-mail : enrique.casalino@aphp.fr

Université Paris-Diderot, Sorbonne-Paris-Cité, EA 7334 « Recherche clinique coordonnée ville-hôpital, Méthodologies et Société (REMES) », F-75018 Paris, France

Study Group for Efficiency and Quality of Emergency Departments and Non-Scheduled Activities Departments, F-75018 Paris, France innovative project. Accreditation visit is one of the tools we can use. A unifying project requires defining shared values among health care workers. The fight against pain is one of the strong values. We need to use the pain management project as a way to review our organizations, evaluate our processes and simplify them, in order to achieve the objectives defined by a specific risk mapping. We present here the experience of our emergency departments for the management of pain of traumatic origin, the methods followed and the improvement tools put in place.

Keywords Pain · Emergency · Continuous quality improvement program $\cdot$ Minor traumatism

\section{Introduction}

L'activité des services d'urgences est en forte progression dans la plupart des pays, dont la France [1]. Des difficultés liées à l'activité, à l'organisation des soins, à la gestion de l'aval, mais aussi à la gestion des processus de soins, sont responsables de la surcharge des urgences ou crowding [2]. La surcharge des urgences a été associée à l'incapacité des services d'urgences à assurer la qualité et la sécurité des soins des patients [3]. De nombreux indicateurs qualité et des indicateurs de performance des services d'urgences ont été proposés dans ce contexte dans l'objectif de garantir la qualité et la sécurité des soins [4]. Nombre de ces indicateurs sont des indicateurs métriques de temps, le principal étant le délai entre l'arrivée et le premier contact avec le médecin et le temps total de passage aux urgences qui a été associé à la survenue de complications, d'effets indésirables et à un surcoût hospitalier [5].

La douleur est le principal motif de consultation aux urgences, et il a été estimé qu'elle concerne sept patients sur dix comme motif de consultation principal ou comme symptôme associé [6]. Sans aucun doute, la prise en charge diagnostique et thérapeutique de la douleur est un point 
central de l'activité des urgences. Les liens entre douleur et qualité, et entre douleur et satisfaction des usagers sont très forts [6,7]. Des études ont montré que la surcharge des urgences est associée à une réduction de l'évaluation de la douleur, de la réévaluation de la douleur et de sa prise en charge thérapeutique [8], mais aussi de la prise en charge de tableaux douloureux sévères comme la douleur thoracique et abdominale [9].

\section{Qualité aux urgences}

Comme tout établissement de santé et service de soins, les services d'urgences sont contraints d'évaluer leurs pratiques de soins. Cette démarche est le plus souvent associée aux processus de certification [10]. Les visites de certification, même si elles peuvent être vécues comme une contrainte par les équipes de soins, permettent de prendre conscience de nos lacunes et de mettre en place des actions d'amélioration de la qualité. Elles sont l'occasion de préparer, de revoir ou de réactualiser nos procédures, de former et de sensibiliser nos équipes à leur utilisation, à définir et à mettre en place des indicateurs qualité ou des traceurs. Il a été démontré que les visites de certification sont associées à une augmentation de la qualité et à une réduction des risques [11]. Certes, les équipes de soins peuvent vivre ces phases hecti- ques comme un épisode fébrile dénoué de sens. C'est à nous managers et responsables du service de donner du sens aux actions menées pour la certification, de les rapprocher de nos valeurs soignantes, de rappeler l'importance de la qualité et la sécurité des soins et du lien étroit avec nos conditions de travail et notre plaisir au travail. Ces améliorations, si elles ne s'intègrent pas dans un projet d'amélioration continue de la qualité, peuvent être de courte durée. Nous devons obtenir l'adhésion des équipes au projet qualité et faire des visites de certification un tremplin permettant de préserver les gains obtenus et poursuivre une amélioration continue de la qualité. La figure 1 présente la différence entre ces deux modèles.

La certification nous impose de préparer une description de nos processus de soins, d'établir une cartographie des risques, de préparer un compte-qualité avec les projets retenus, d'évaluer nos pratiques exigibles prioritaires, d'établir des indicateurs qualité, d'évaluer nos pratiques professionnelles et d'analyser les parcours de soins transversaux des patients par l'analyse des patients traceurs. Chacun de ses éléments est fondamental dans un projet d'optimisation des parcours de soins, d'amélioration de la qualité et de la sécurité des soins, d'amélioration de la satisfaction des usagers, d'amélioration des conditions de travail des équipes et de la satisfaction et le plaisir au travail des personnels des urgences.

\section{Un pic d'optimisation et de respect \\ des procédures, un coup de fièvre pour nos équipes!}

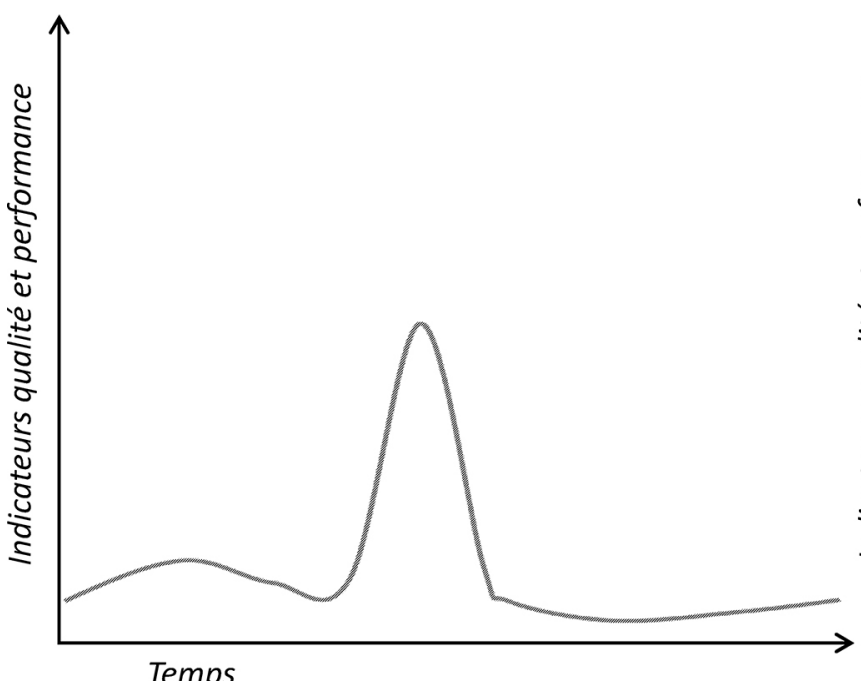

\section{La certification doit être un puissant moteur d'amélioration continue de la qualité !}

Fig. 1 Comparaison des interventions ponctuelles et d'un projet d'amélioration continue de la qualité

\section{Un processus continu d'amélioration et une culture d'efficacité mènent les équipes à une amélioration}

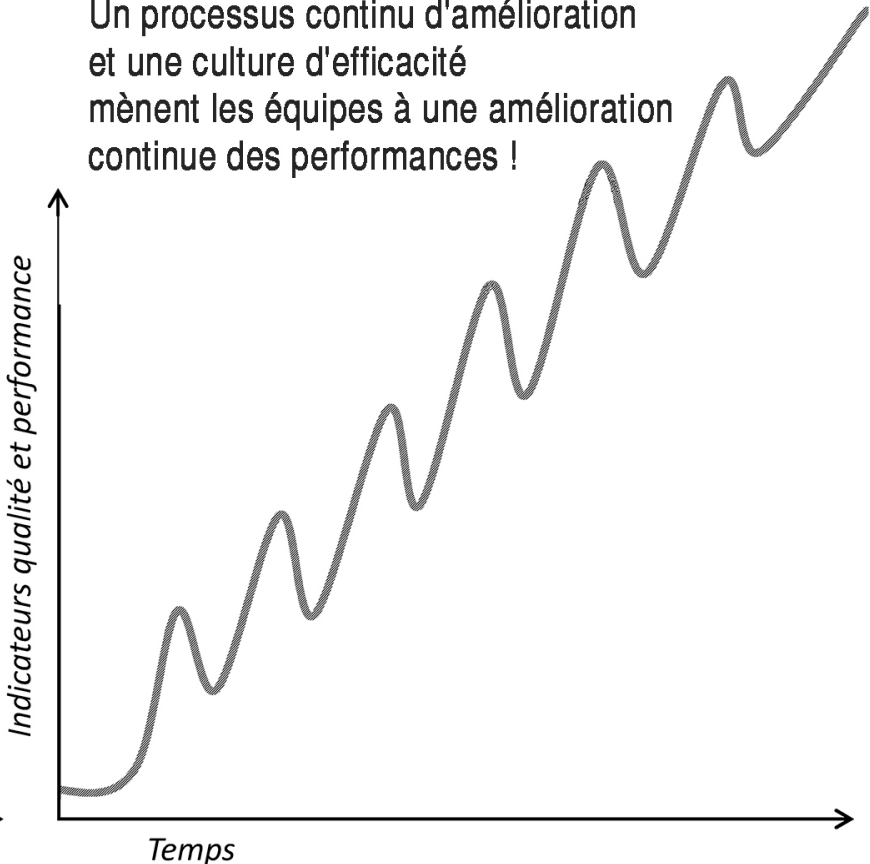

Temps 


\section{Définir les valeurs et les objectifs}

La certification est un outil, elle n'est pas une fin en soi. Il en va de même pour la qualité et la performance : elles sont le chemin pour atteindre nos objectifs qui reposent sur nos valeurs soignantes. Il est indispensable au moment de concevoir un projet d'amélioration continue de la qualité [12] de définir les valeurs qui animent cette stratégie. Ces valeurs doivent être partagées par les équipes. Ces valeurs, dans le monde de la santé, sont le plus souvent des valeurs soignantes, inspirées du patients-centered care [13] : le patient doit être au centre de toutes nos organisations, nos préoccupations, nos objectifs.

À titre d'exemple, aux SAUs Bichat et Beaujon, nos valeurs ont été définies comme deux piliers :

- le patient : la lutte contre la maltraitance et le développement de la bientraitance des patients [14];

- les équipes de soins : lutter contre le burnout, optimiser les conditions de travail et développer le plaisir au travail [15].

Définir des objectifs à court, moyen et long termes est essentiel pour pouvoir conduire un projet d'amélioration continue de la qualité. Dans notre expérience, la définition par l'équipe d'encadrement d'une vision du service idéal vers lequel nous voulons avancer est indispensable. Cette vision idéale permet de construire le chemin, les préalables et les jalons sur lesquels nous devons avancer pour atteindre cette vision. La diminution des délais d'attente IAO et médecin ne sont que des jalons intermédiaires, justifiés par la nécessité de garantir des places vacantes pour accueillir des patients arrivant avec une installation directe par les IAO, ces deux jalons nous permettant d'atteindre l'objectif final « aucun patient dans les couloirs des urgences ». Cet objectif, fondé sur la bientraitance des patients et les conditions de travail des équipes, a reposé sur une phrase qui a fédéré les équipes : « un patient dans les couloirs c'est de la maltraitance ». Cette valeur et cet objectif ont été partagés par tous les agents, ils sont précisés dès les entretiens d'embauche et dans les formations d'intégration, et rappelés lors de chaque transmission [16].

\section{La douleur : moteur de l'amélioration de la qualité et de la performance aux urgences}

Certaines thématiques peuvent avoir une valeur exemplaire et fédératrice. Dans l'expérience du SAU Bichat, la violence [17] et la douleur ont été des éléments fondateurs du projet qualité [16]. La douleur est dans l'imaginaire de la population, mais aussi des soignants, quelque chose d'intense comme expérience et traduit la sensibilité des soignants. Qui n'a pas entendu une personne raconter révoltée « j'avais mal et personne ne s'est occupé de moi !", ou «j'avais horriblement mal, et personne ne me croyais !», ou encore «j'avais mal, on ne m'a rien demandé ni rien donné !», ou pire « en fait, personne n'avait rien à faire de ma douleur ni de moi ». Les valeurs du service, lutte contre la maltraitance, respect de la dignité du patient et bientraitance du patient, se retrouvent parfaitement dans une stratégie ambitieuse de prise en charge de la douleur. Soulager les patients est une obligation déontologique et légale [18], un enjeu de santé publique [19] et un objectif qualité prioritaire national [20]. Le lien entre satisfaction des patients et prise en charge de la douleur a été démontré, l'évaluation de la douleur et la prise en charge thérapeutique rapide ont été associées à la satisfaction, et la prise en charge efficace de la douleur a été associée à la réduction du temps de séjour aux urgences [7,21-23]. Pour toutes ces raisons, l'évaluation des modalités de prise en charge de la douleur aux urgences est un des critères exigibles de la démarche de certification [10].

\section{Projet d'amélioration de la prise en charge de la douleur}

Un projet d'amélioration de la prise en charge de la douleur doit suivre les étapes habituelles d'un projet d'amélioration continue de la qualité. Dans un objectif pédagogique, nous prendrons l'exemple du projet d'amélioration de la qualité de la prise en charge de la douleur traumatique en cours d'implémentation aux SAUs Bichat et Beaujon.

Les objectifs sont en accord avec nos valeurs, bientraitance des patients et meilleures conditions de travail pour nos équipes. Ils doivent être simples et partagés : évaluation immédiate et systématique de la douleur dès l'arrivée, réduction du délai entre l'arrivée du patient et l'administration de l'antalgique, prévention de la douleur induite par le transport et par la mobilisation des membres lors des examens de radiologie, réduction du risque d'oligoanalgésie définie comme une prise en charge insuffisante de la douleur [24,25], évaluation de la douleur au cours du séjour aux urgences et avant la sortie du SAU, augmenter la satisfaction du patient et garantir la continuité des soins.

L'analyse des processus de soins doit permettre d'identifier les risques à chacune des étapes du processus de soins, de définir des objectifs pour chacun des processus et de proposer des stratégies de simplification des processus. La figure 2 présente l'expérience des SAUs Bichat et Beaujon dans le cadre de la démarche d'amélioration de la prise en charge des patients avec des traumatismes. Nous pouvons observer que parmi les risques que nous avons identifiés le délai entre l'arrivée et la prise en charge effective de la douleur était très long : il fallait attendre la prise en charge par le médecin, la prescription d'un antalgique, l'intervention de l'IDE pour l'administrer, le plus souvent par voie 
intraveineuse, ce qui entraîne d'autres risques pour le patient et pour le personnel de soins ; et en radiologie, l'absence de prise en charge du risque de douleur induite par des mobilisations pour la réalisation de l'examen de radiologie.

La stratégie d'amélioration est ainsi axée sur la priorisation de l'accueil par l'IAO, la prescription anticipée d'antalgiques par l'IAO, le triage en fonction de l'intensité de la douleur, l'installation immédiate des patients avec une indication de morphine en titration et l'orientation directe en radiologie par prescription anticipée des examens de radiologie des membres pour les autres patients. Nous observons également que l'approche qualité totale permet de renforcer la prévention de la douleur induite en radiologie par les mouvements des membres, mais aussi dans les secteurs de soins lors d'actes ou de gestes de réduction ou d'immobilisation, la réévaluation de la douleur en secteur de soins et avant la sortie, de renforcer le rôle du médecin et des IDEs des secteurs de soins dans la prise en charge de la douleur en fonction des réévaluations réalisées avec réduction du risque d'oligoanalgésie, de souligner l'importance des ordonnances de sortie adaptées à la typologie de la douleur et de garantir la filière de soins posturgence proposée au patient.

Cette approche devrait réduire les périodes d'attente inutile, notamment par une installation immédiate en box, de réduire le délai entre l'arrivée et l'administration de l'antalgique par la prescription anticipée des antalgiques par l'IAO, et de réduire le délai entre l'arrivée et l'examen radiologique par une prescription anticipée par l'IAO des examens radiologiques de traumatismes des membres avec orientation directe en radiologie. La réduction des intervalles de temps est un moyen, mais pas l'objectif. La stratégie de réduction de la douleur induite lors des mobilisations en radiologie a été une préoccupation constante de nos équipes dans le cadre de la lutte contre la maltraitance des patients.

La mise à disposition d'un antalgique avec un dispositif autoadministré et un délai d'action très court pour les patients avec des douleurs modérées à sévères pourrait être un outil thérapeutique permettant une réduction rapide de

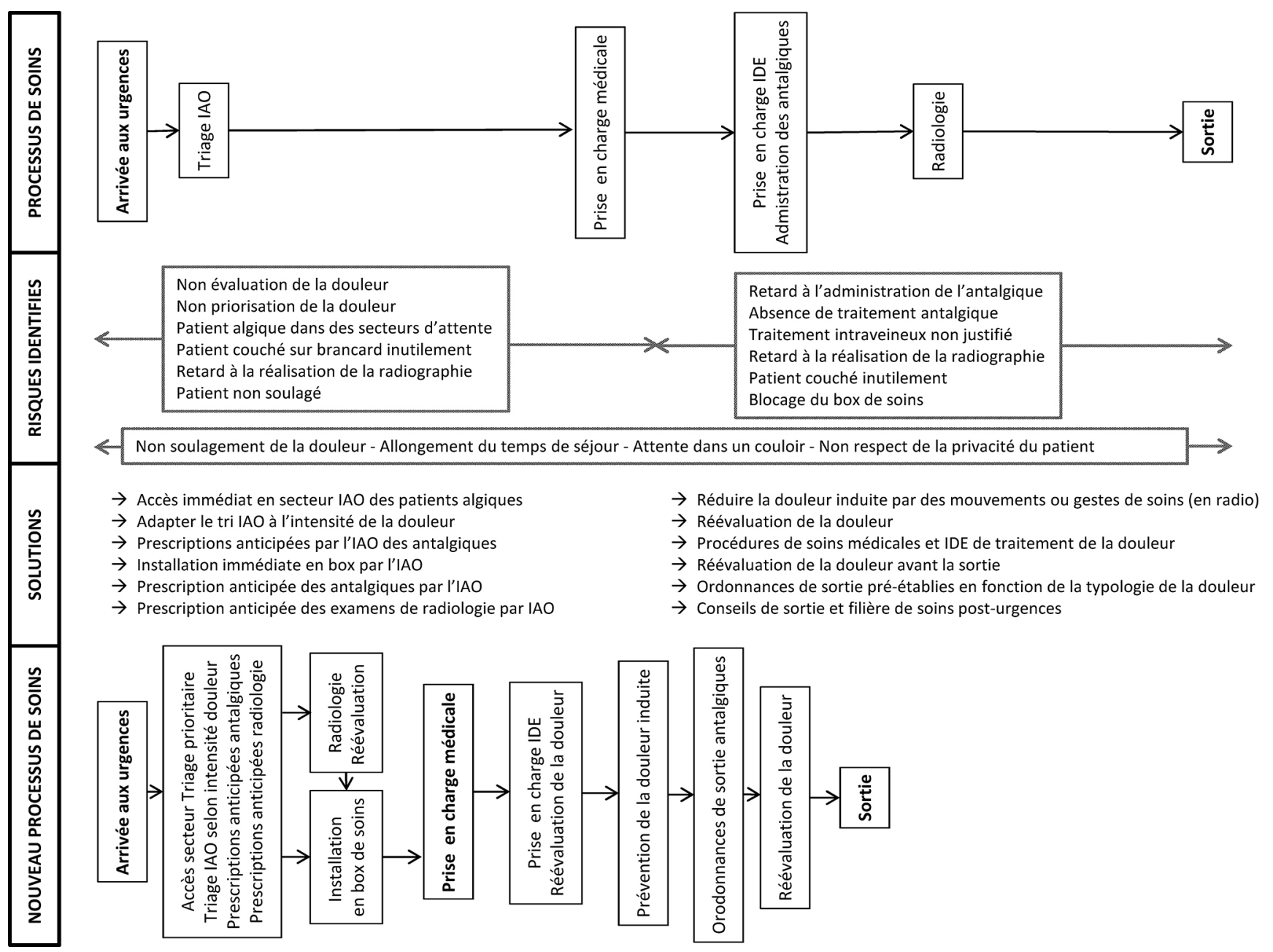

Fig. 2 Modèle d'évaluation des processus et plan des interventions à l'amélioration de la qualité de la prise en charge de la douleur d'origine traumatique IAO : infirmier(ère) accueil et organisation ; IDE : infirmier(ère) diplomé(e) d'État 
l'intensité de la douleur dans les toutes premières étapes de prise en charge aux urgences et de la douleur induite en radiologie par les mouvements des membres.

Ces stratégies d'amélioration de la qualité incluant de nouveaux outils et organisations se heurtent à des difficultés de mise en place et d'évaluation méthodologiques complexes. Le renouvellement des personnels des urgences, des aspects réglementaires concernant les prescriptions anticipées, la surcharge des urgences ne sont que quelques éléments à prendre en compte.

\section{Traceurs et indicateurs de performance}

Nous avons proposé et nous débutons la mesure de l'intensité de la douleur lors des premières étapes de soins, et des intervalles de temps entre l'arrivée du patient et l'administration du premier antalgique, la prescription et la réalisation de la radiographie. Ces intervalles traduisent la performance du service à organiser un chemin clinique et une articulation avec des partenaires extérieurs au service. Nous devons également mesurer le pourcentage de patients ayant eu une évaluation de la douleur à l'IAO, lors de la radiographie, au retour dans le secteur de soins et avant la sortie, mais aussi le pourcentage de patients avec une ordonnance de sortie avec des antalgiques. Un autre indicateur doit être le pourcentage de patients adressés dans une consultation adaptée à leur motif de consultation. Enfin, nous devrons évaluer la satisfaction du patient et la satisfaction des personnels du service par cette nouvelle procédure.

\section{Conclusion}

La qualité est un impératif pour nos services d'urgences comme pour tous nos établissements. Néanmoins, elle ne doit pas être vécue comme une contrainte. Nous sommes convaincus que la certification comme les démarches d'amélioration continue de la qualité aux urgences sont des occasions de revoir nos processus de soins et de proposer des améliorations dans nos organisations et dans nos processus de soins permettant de garantir la sécurité des soins de nos patients et les conditions de travail de nos équipes. L'optimisation de la prise en charge de la douleur est un projet capable de mobiliser les équipes de soins et de mettre en place des projets de changement innovants et motivants pour les équipes.

Liens d'intérêts : missions ponctuelles de conseil pour Mundipharma.

\section{Références}

1. Jiang JH, Weiss AJ, Barrett M, Agency for Healthcare Research and Quality (2017). Healthcare Cost and Utilization Project (HCUP) Characteristics of emergency department visits for super-utilizers by payer, 2014. Statistical Brief \#221. https:// www.ncbi.nlm.nih.gov/books/NBK442038/pdf/Bookshelf_NBK442038.pdf (Dernier accès le 10 février 2018)

2. Wang H, Ojha RP, Robinson RD, et al (2017) Optimal measurement interval for emergency department crowding estimation tools. Ann Emerg Med 70:632-9.e4

3. Zhou X, Zhang Z, Li J, et al (2018) The impact of emergency department crowding on outcomes, other aspects should not be ignored. Am J Emerg Med In press pii: S07356757(17)30538-7 http://www.sciencedirect.com/science/article/ pii/S0196064417304328?via\%3Dihub (Dernier accès le 10 février 2018)

4. Madsen MM, Eiset AH, Mackenhauer J, et al (2016) Selection of quality indicators for hospital-based emergency care in Denmark, informed by a modified-Delphi process. Scand J Trauma Resusc Emerg Med 24:11

5. Pines JM, Hilton JA, Weber EJ, et al (2011) International perspectives on emergency department crowding. Acad Emerg Med 18:1358-70

6. Todd KH, Ducharme J, Choiniere M, et al (2007) Pain in the emergency department: results of the Pain and Emergency Medicine Initiative (PEMI) multicenter study. J Pain 8:460-6

7. Chang AM, Lin A, Fu R, et al (2017) Associations of emergency department length of stay with publicly reported quality-of-care measures. Acad Emerg Med 24:246-50

8. Kone V, Lecomte F, Randriamanana D, et al (2016) Impact of a pilot team on patients' pain reduction and satisfaction in an emergency department: a before-and-after observational study. Rev Epidemiol Sante Publique 64:59-66

9. Pines JM, Shofer FS, Isserman JA, et al (2010) The effect of emergency department crowding on analgesia in patients with back pain in two hospitals. Acad Emerg Med 17:276-83

10. Smith B, Bouchoucha S, Watt E (2016) "Care in a chair" - the impact of an overcrowded emergency department on the time to treatment and length of stay of self-presenting patients with abdominal pain. Int Emerg Nurs 29:9-14

11. Haute Autorité de santé (HAS) (2016) Certification des établissements de santé. https://www.has-sante.fr/portail/jcms/fc_1249882/ $\mathrm{fr} /$ certification-des-etablissements-de-sante (Dernier accès le 10 février 2018)

12. Barnett ML, Olenski AR, Jena AB (2017) Patient mortality during unannounced accreditation surveys at US hospitals. JAMA Intern Med 177:693-700

13. The National Learning Consortium. Health Information Technology Research Center (HITRC) (2013) Continuous quality improvement (CQI) strategies to optimize your practice. https://www. healthit.gov/sites/default/files/tools/nlc_continuousqualityimprovementprimer.pdf (Dernier accès le 10 février 2018)

14. McConnell D, McCance T, Melby V (2015) Exploring personcentredness in emergency departments: a literature review. Int Emerg Nurs 26:38-46

15. Casalino E (2014) Les urgences : du constat de la maltraitance à un projet qualité basé sur la bientraitance. In: Schmitt M (ed) Bientraitance et qualité de vie, outils et retour d'expériences. Elsevier-Masson Ed., Paris, pp 213-23

16. Boyer F (2017) Le plaisir au travail. Innovation managériale. http://www.innovationmanageriale.com/concepts/de-la-gestiondu-savoir-faire-au-management-de-laimer-faire/ (Dernier accès le 10 février 2018) 
17. Casalino E, Choquet C, Wargon M, et al (2015) Continuous quality improvement program, based on lean concepts, allows emptying of emergency department corridors. Am J Accountable Care 12:15. http://www.ajmc.com/journals/ajac/2015/2015-vol3n4/Continuous-Quality-Improvement-Program-Based-on-LeanConcepts (Dernier accès le 10 février 2018)

18. Casalino E, Choquet C, Thomas S, et al (2015) La violence dans les services d'urgences : évaluation d'une politique de réduction de la violence dans un service d'urgences parisien. Ann Fr Med Urgence 5:226-37

19. République française (2002) Loi $\mathrm{n}^{\mathrm{o}} 2002-303 \mathrm{du} 4$ mars 2002 relative aux droits des malades et à la qualité du système de santé. https://www.legifrance.gouv.fr/affichTexte.do?cidTexte=JORFTEXT000000227015 (Dernier accès le 10 février 2018)

20. Ministère de la Santé et des Solidarités (2010) Plan d'amélioration de la prise en charge de la douleur 2006-2010. Mise à jour décembre 2009. http://social-sante.gouv.fr/IMG/pdf/Plan_d_amelioration_de_la_prise_en_charge_de_la_douleur_2006-2010_.pdf. (Dernier accès le 10 février 2018)
21. Haute Autorité de santé (HAS) (2014) Manuel de certification des établissements de santé V2010 — édition janvier 2014 https://www.has-sante.fr/portail/jcms/c_1732464/fr/manuel-decertification-des-etablissements-de-sante-v2010-edition-janvier2014 (Dernier accès le 10 février 2018)

22. Karwowski-Soulie F, Lessenot-Tcherny S, Lamarche-Vadel A, et al (2006) Pain in an emergency department: an audit. Eur J Emerg Med 13:218-24

23. Pierik JG, IJzerman MJ, Gaakeer MI, et al (2015) Pain management in the emergency chain: the use and effectiveness of pain management in patients with acute musculoskeletal pain. Pain Med 16:970-84

24. Pierik JGJ, IJzerman MJ, Gaakeer MI, et al (2017) Painful discrimination in the emergency department: risk factors for underassessment of patients' pain by nurses. J Emerg Nurs 43:228-38

25. Boccard E, Adnet F, Gueugniaud PY, et al (2011) Pain management in adult patients in emergency care units in France in 2010. Ann Fr Med Urgence 1:312-9 